

\title{
Nada es lo que parece: una reflexión sobre las visiones deformadas de la ciencia
}

- Nothing is What it Seems: Reflecting on Science Distorted Visions

- Nada é o que parece: uma reflexão sobre as visões distorcidas da ciência

\section{Resumen}

En este artículo se lleva a cabo una reflexión acerca de las denominadas visiones deformadas de la ciencia, desde la perspectiva educativa y epistemológica de un estudiante del Máster Universitario en Profesor de Educación Secundaria Obligatoria, Formación Profesional y Enseñanza de Idiomas. Se pretende entonces con este texto presentar algunas ideas en torno a ocho distorsiones identificadas por la investigación en didáctica de las ciencias, las cuales se apoyan mutuamente formando un marco conceptual que obtura la posibilidad de alcanzar una genuina alfabetización científica. Las prácticas escolares más tradicionales continúan, todavía hoy, transmitiendo una imagen empobrecida, mitificada, desencantada e ingenua de las disciplinas científicas, de sus objetivos, de sus métodos, de la evolución y desarrollo de sus conocimientos; una perspectiva que enmarcará su verdadera naturaleza al presentarlas como un saber esotérico, elitista, inútil, ahistórico, descontextualizado y excesivamente analítico. Se postula, a modo de conclusión, la necesidad de introducir en la enseñanza la educación no formal como herramienta que contribuye a erradicar dichas visiones deformadas.

\section{Palabras clave:}

ciencia; didáctica de las ciencias; educación; naturaleza de la ciencia; visiones deformadas

\section{Jorge Martín-García*}

Máster Universitario en Profesor de educación secundaria obligatoria, formación profesional y enseñanza de idiomas, Universidad de Salamanca; Departamento de Didácticas Específicas, Universidad de Zaragoza, Zaragoza. Correo electrónico: araujo@unizar.es. Código Orcid: https:// orcid.org/0000-0002-2247-0826 


\section{Abstract}

This article reflects on the distorted views of science from an educational and epistemological perspective of a student of the master's degree in Teacher of Compulsory Secondary Education, Vocational Training and Language Teaching. It is intended then with this text to present some ideas around eight distortions identified by research in science didactics, which mutually support each other forming a conceptual framework that blocks the possibility of achieving a genuine scientific literacy. The most traditional school practices continue, even today, transmitting an impoverished, mythologized, disenchanted and naive image of the scientific disciplines, of their objectives, of their methods, of the evolution and development of their knowledge; a perspective that will frame their true nature by presenting them as esoteric, elitist, useless, ahistorical, decontextualized and excessively analytical knowledge. In conclusion, the need to introduce non-formal education into teaching as a tool that contributes to eradicating these distorted views is postulated.

Keywords:

science; science didactics; education; nature of science; distorted views

\section{Resumo}

Neste artigo realizou-se uma reflexão sobre as visões distorcidas da Ciência na perspectiva educacional e epistemológica de uma aluna do Mestrado Universitário em Ensino Médio Obrigatório, Formação Profissional e Ensino de Línguas. Pretende-se apresentar algumas ideias em torno dessas oito distorções identificadas pela pesquisa em didática da ciência, que se apoiam mutuamente, formando um arcabouço conceitual que bloqueia a possibilidade de se atingir um letramento científico genuíno. As práticas escolares mais tradicionais continuam, ainda hoje, a transmitir uma imagem empobrecida, mitificada, desencantada e ingénua das disciplinas científicas, dos seus objectivos, dos seus métodos, da evolução e do desenvolvimento dos seus saberes; uma perspectiva que enquadra sua verdadeira natureza e os apresenta como conhecimentos esotéricos, elitistas, inúteis, a-históricos, descontextualizados e excessivamente analíticos. Em conclusão, postula-se a necessidade de inserir a educação não formal no ensino como ferramenta que contribua para erradicar essas visões distorcidas.

\section{Palavras-chave:}

ciência; didática da ciência; educação; natureza da ciência; visões distorcidas 


\section{Introducción}

La ciencia y la tecnología, desde siempre, han desempeñado un rol esencial en la evolución social, al expandir las fronteras del conocimiento han contribuido al exponencial incremento del nivel y la calidad de vida que se ha producido en las últimas décadas. En esta coyuntura, la educación científico-tecnológica se convierte en una necesidad básica (Gil et ál., 2005), un principio clave, en el progreso de los individuos y las civilizaciones.

Desafortunadamente, ese protagonismo que se viene señalando, no ha venido acompañado de una mayor comprensión de esta a nivel académico ni a nivel social. Niños, adolescentes, adultos e incluso los propios investigadores tienen imágenes de la ciencia que responden a una colección de tópicos y estereotipos negativos y perniciosos, que se han abierto camino en el seno de la sociedad hasta quedar enquistados en la mente de la mayoría, donde han comenzado a aceptarse socialmente como verdaderos, alcanzando un estatus cultural pero alterando, enmascarando y distorsionando, notablemente, las percepciones de los individuos sobre la verdadera naturaleza de la actividad científica.

Estas visiones, que se transmiten muchas veces implícitamente desde la escuela, se traducen en lo que se ha denominado visiones deformadas de la ciencia (Chade, 2014; Fernández et ál., 2002; Gallego, 2007; López y Furió, 2017). No se trata de una serie de ideas aisladas, disonantes, desligadas y autónomas, sino que componen un cúmulo de deformaciones que se apoyan recíprocamente, sustentadas para engendrar un marco conceptual coherente, con una cierta cohesión, un esquema alternativo relativamente integrado, que genera desinterés y que justifica, en gran medida, tanto el desprecio que suscita la ciencia en nuestros jóvenes (Segarra et ál., 2017), como las elevadas tasas de fracaso escolar que asolan las materias afines a ella y que han llegado a considerarse completamente normales.

\section{Las ocho visiones deformadas de la ciencia que se transmiten desde la enseñanza}

Las diferentes visiones sobre las que se va a desarrollar el artículo fueron inicialmente propuestas y desarrolladas por Fernández et ál. (2002), aunque posteriormente otros muchos autores se han apoyado en ellas.

\section{Una imagen empirista y ateórica de la ciencia}

Cuantiosas son las discrepancias que pueden ser detectadas entre la cosmovisión de la ciencia que se contempla desde las tendencias epistemológicas más contemporáneas y aquellas que podemos descubrir en cualquier aula de secundaria, donde imperan nociones caracterizadas por un empirismo extremo, exagerado incluso.

La mayoría de los estudiantes cree que la ciencia se reduce a descubrir una realidad preexistente, a lo que contribuye sobremanera esta concepción, que ensalza la observación y la experimentación neutras (Fernández et ál., 2002), no contaminadas por ideas apriorísticas que no se apoyan en la experiencia (Gallego, 2007), constituyendo lo que, en ocasiones, se ha denominado "empirismo ingenuo". El conocimiento científico, en esta visión se genera mediante inducción, a partir de datos teóricamente neutros, recabados por los dos elementos anteriores (Chade, 2014).

No se tiene en cuenta, por lo tanto, el sustento teórico que ha de guiar el proceso, ni los supuestos de partida que lo motivan, relegando la hipótesis a un segundo plano

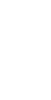


y obviando su papel primordial, focalizador; suprimiendo la influencia de la creación de cuerpos coherentes de conocimientos en la orientación del proceso de investigación.

Tampoco se muestra cómo el desarrollo científico parte, inicialmente, de una situación problemática amplia y confusa, que no es nítida y cómo, consecuentemente, es necesario primero definir claramente el problema, de forma precisa, diseccionándolo y realizando las simplificaciones que sean pertinentes para poder abordarlo poco a poco y en la medida en que los conocimientos, las técnicas y la tecnología del momento permitan hacerlo, modelizando la situación para trabajar más fácilmente con ella y marcando una serie de objetivos a cumplir (Fernández et ál., 2005).

Pertinentes o éticas, porque se ha de considerar hasta qué punto es necesario respetar la totalidad del objeto de estudio, su complejidad intrínseca. Plantearse si realmente la disección de un fenómeno permite una adecuada aproximación a él, que asegure una comprensión profunda del mismo y no una mera visión superficial que traiga consigo la pérdida de informaciones relevantes, como puede ser la dinámica de interacción entre los distintos factores o elementos que lo constituyen; o la emergencia de propiedades que no pueden ser explicadas desde las características de cada uno de sus elementos constitutivos.

Solo entonces es posible dar paso a la realización de pruebas y experimentos, pues, aunque es cierto que la obtención de datos empíricos en condiciones estrictamente controladas ocupa un papel central en el desarrollo científico (Borda y Erazo, 2010), no es menos cierto que dichos datos, por sí mismos, carecen de sentido y solo lo adquieren cuando son considerados dentro de un entramado teórico, en relación a las hipótesis que se pretenden contrastar y al diseño experimental empleado en cada caso (Fernández et ál., 2005).

Seguramente estemos ante una de las deformaciones más arraigadas en la enseñanza secundaria y su razón de ser podría, perfectamente, ser el hecho de que, a pesar de la importancia "de palabra" que se le da a la observación y la experimentación, a la hora de la verdad, en general, las clases continúan teniendo un carácter eminentemente expositivo de transmisión de contenidos, sin oportunidades apenas de trabajo experimental, el cual adquiere para el adolescente un halo de misticismo y atractivo, convertido en el anhelo de una revolución pendiente.

\section{Una visión rígida, algorítmica, exacta e infalible de la actividad científica}

Por la que se concibe el trabajo científico como un conjunto rígido, inamovible, de etapas, que se ha de seguir de forma mecánica, el archiconocido "método científico" (Gil y Vilches, 2001). Una prescripción, un algoritmo infalible, de validez universal e independiente del contexto que garantiza la exactitud y la 
objetividad de los resultados, una receta para alcanzar el conocimiento verdadero, la auténtica esencia de una realidad objetiva que espera que la descubramos.

Son concepciones que resaltan el rigor y la exactitud de los resultados obtenidos, pero ignorando, o incluso rechazando todo aquello que implique el esfuerzo imaginativo del científico, la invención, la creatividad, la duda (Gil y Vilches, 2001), donde aparece, latente, la idea de que solo a través de la rigurosidad metodológica puede llegarse al conocimiento y que este solo es válido si se han respetado las pautas marcadas al pie de la letra, cayendo en la trampa de un absurdo absolutismo metodológico (Acevedo, 2000).

Sin embargo, es importante remarcar el papel que tiene el pensamiento divergente en la investigación (Fernández et ál., 2005). Aunque es innegable que los equipos, los procedimientos y las pruebas son de la máxima utilidad y unos aliados de incalculable valor para el científico, de nada sirven sin la intuición del investigador (Moreno, 2006), sin su imaginación, creatividad e inventiva. El desarrollo científico precisa de la creación de conceptos, teorías y postulados que posteriormente sean contrastados con la experiencia, así como de modelos que puedan ser visualizados y alterados en nuestra imaginación para trabajar con ellos y analizarlos. Es a nosotros, los docentes, a quienes atañe remarcar esa naturaleza híbrida de la ciencia (Arraiz y Sabirón, 2012), colmada de precisión, de medidas, estándares, datos y resultados, de consecuencias y rendimientos, pero nunca descargada de ideas, de iniciativa, de fantasía y originalidad, de encanto y humanidad, sobre todo de humanidad.

No existe "el" método científico, no hay una receta predefinida sobre cómo hacer ciencia ni un único procedimiento estándar aceptado unánime y universalmente por la comunidad científica al que ceñirse y que haya funcionado a lo largo de la historia (Chade, 2014). Como la propia ciencia, el método es esclavo de su contexto, que lo convierte en un elemento histórico-concreto (Cabot, 2014), por lo que, a medida que hemos ido avanzando en el estudio y la comprensión de los fenómenos, en ese proceso ininterrumpido de dominio del entorno; su significado, características e implicaciones han ido cambiando, diluyéndose en las tendencias epistemológicas y las prácticas hegemónicas de cada momento.

El progresivo desarrollo de las disciplinas, insisto, ha derivado en diferentes tradiciones que han determinado la forma de abordar los problemas científicos que pueden plantearse; sin embargo, al final es la combinación de todas ellas la que de verdad ha marcado el avance de la ciencia.

En los tiempos que corren, donde ya se ha hecho patente que la realidad se encuentra por encima de toda demarcación disciplinar, tendremos que plantearnos una nueva forma de entender y organizar el conocimiento; nuestro objeto de estudio ya no puede circunscribirse a una única tradición disciplinar, sino que ha de ser reconsiderado desde una aproximación que dé cabida a su complejidad. Estamos entrando en la era de la Complejidad, donde la transdisciplinariedad y la multirreferencialidad demandan la adopción de una posición filosófica que dé un paso atrás para analizar cómo diferentes paradigmas pueden moldear la construcción de conocimiento (Berbegal, 2015).

Que deberá tener en cuenta las tesis y perspectivas antagónicas, en tanto que apostar por una sola de ellas resulta insuficiente y se traduce en la pérdida de la parte de verdad. Al abrazar el paradigma de la Complejidad estaremos reconociendo y reivindicando pre- 
cisamente esa contradicción, y, por tanto, idearemos nuevas formas de trabajar que sean coherentes y encajen con esta nueva perspectiva epistemológica.

Contra el estereotipo de rigidez absoluta, de orden escrupuloso y minuciosa secuenciación de procedimientos, emerge un método científico caracterizado por su diversidad de formas, convertido en una pauta flexible y amplia, permeable, que se adapta a las particularidades del campo y del momento, que se encuadra, encontrando su fundamento en un corpus teórico coherente y que no es ajeno a ni a los factores subjetivos ni a la ética. Superada la antigua visión que lo reducía a una simple receta se abre paso una nueva forma de concebirlo, que lo entiende como una estrategia global de enfrentamiento al conocimiento del mundo, encaminada a la penetrar y transformar la realidad (Cabot, 2014).

La actividad científica es un proceso racional, de continuo contraste de los hechos con la naturaleza, que procede gradualmente, marcada por el hecho de que de que los resultados obtenidos son aproximaciones cada vez mejores a la realidad a la que se aplican, pero que nunca constituyen un ajuste definitivo, exacto o invariable, sometido a un continuo proceso de autocorrección, de identificación de fallos y de mejora de respuestas siempre sujeto al surgimiento de nuevas preguntas, por lo que se trata más bien de un ciclo, un intrincado laberinto en el que cada disciplina tiene sus particularidades y cada investigador sus manías, su propia idiosincrasia, que determinará cómo va a abordar el estudio del fenómeno que tiene ante sí.

La caza de errores, de datos que no encajan, o de anomalías que atentan contra las bases del paradigma establecido son motivo de crisis; pero, al mismo tiempo, constituyen el fundamento último del progreso del conocimiento científico, son el motor que impulsa su avance y su mayor aliciente porque llevan a la continua modificación de las hipótesis, cuna del surgimiento de los saberes. Así, los razonamientos no se hacen en términos de certezas absolutas, sino en términos de suposiciones, apoyadas en una colección de evidencias y conocimientos adquiridos, pero que son concebidas, como tentativas de respuesta provisionales (Fernández et ál., 2005), e incluso si la hipótesis sea aceptada como paradigma de una disciplina no se constituye en sí misma una verdad, sino un conocimiento temporal, que sencillamente no ha podido ser refutado todavía y que llegado el momento volverá a ser puesto a prueba y corregido si así se requiere, para dar de nuevo comienzo al ciclo.

En la naturaleza de la ciencia no hay ya lugar para constataciones y falsaciones definitivas (Borda y Erazo, 2010), ya no existen verificaciones o demostraciones concluyentes, terminantes o inapelables y, de hecho, la actividad científica se ha caracterizado cada vez más, por la ineludible renuncia a la certidumbre y la innegable necesidad de aceptar que la descripción del mundo que nos rodea ha de hacerse en términos probabilísticos, estadísticos, haciendo de la duda su emblema, de la posibilidad su bandera, del dilema de su himno. 
El principio de incertidumbre de Heisenberg, la hipótesis de Planck, la dualidad onda corpúsculo, el nacimiento e impresionante desarrollo de la mecánica cuántica, con sus asombrosas consecuencias, el quark, el gluon, - la cromodinámica cuántica han puesto en duda la propia noción de realidad, su esencia, acabando con la dictadura del principio del orden; han enterrado el determinismo clásico, imponiendo restricciones fundamentales a lo que podemos conocer, comprender e incluso percibir, a lo que podemos medir y cuantificar, a lo que podemos analizar y han desencadenado que tanto la aleatoriedad como el azar hayan de erigirse principios primordiales en nuestra travesía hacia la intelección de nuestro entorno y hasta de nosotros mismos.

Duda, confusión, incertidumbre, inseguridad, paradojas, contradicciones, titubeos, o perplejidad no son verdugos del saber, estertores en el deceso del conocimiento, sino más bien adalides del cambio que ha de modernizar la ciencia y la tecnología, precursores de una nueva forma de pensar, pioneros en el tránsito hacia la Complejidad; la vanguardia que alerta de la imposibilidad, insisto, de dominar por completo, de una manera precisa, quirúrgica, cirujana, certera, todas las fases, condiciones estados o disposiciones que un fenómeno es susceptible de exhibir o exteriorizar (Berbegal, 2015), apremiándonos a reconocer y afrontar que la realidad es ambivalente, polifacética, heterogénea, que en ella existen infinidad de niveles, donde no tienen cabida las aproximaciones diseccionistas y hay que arriesgarse abrazando perspectivas más holísticas, que no despedacen el objeto de estudio, que no rompan la dinámica de interacción entre sus distintas partes, las interacciones entre sus componentes.

Una orientación que admita que el pleno conocimiento de las partes no implica el del todo, una cosmovisión nueva, desarrollada desde una lógica difusa, borrosa, reflejo de la naturaleza imprecisa, confusa, velada del universo.

\section{Una concepción aproblemática y ahistórica de la ciencia}

En los enfoques tradicionales de enseñanza no suele hacerse referencia al proceso de producción de conocimientos científicos a lo largo de las diferentes etapas históricas. La escuela se limita a exponer unos conceptos ya elaborados, sin dotarles del carácter hipotético y evolutivo que tienen. Se presentan, eso sí, dentro de una estructura lógica en el amplio marco de la disciplina a la que pertenecen, pero sin tener en cuenta los problemas o modelos que existieron en su origen, que sentaron las bases para su construcción y evolución, ni tampoco las dificultades que tuvieron que ser superadas.

Al mismo tiempo, se introducen las teorías hegemónicas sin hacer referencia a aquellas con las que compitieron por la preeminencia, como si no hubieran existido alternativas factibles; y, cuando se mencionan estas, es para mostrar que el punto de vista "correcto" triunfó y pudo imponerse a los "incorrectos". Se evita mostrar cómo dos hipótesis o leyes distintas convivieron, compitiendo, durante espacios de tiempo prolongados, hasta que un cambio en las condiciones tuvo como consecuencia que una de ellas tuviera que ser descartada.

Solemos olvidar que los contenidos que enseñamos hoy son las soluciones a las necesidades epistemológicas y conceptuales de ayer, que toda investigación responde a un problema, una cuestión íntimamente vinculada a las necesidades humanas (García et ál., 2003). De ahí la importancia manifiesta que tiene el análisis de la evolución histórica de los conceptos y cómo se han ido articulando en cuerpos coherentes de saberes asociados que, 
además, se han construido dentro de un contexto humano, influenciados por unas condiciones sociales, económicas filosóficas y epistemológicas definidas; las cuales han determinado, directa o indirectamente, las cuestiones planteadas y el enfoque que se le ha dado en cada momento (Campanario, 1998).

Los individuos somos científicos desde la cuna, la ciencia nos ha acompañado a lo largo de las edades y durante todos y cada uno de estos años de sincera y noble adhesión, el desarrollo científico, se ha producido siempre en entornos específicos, de la mano de personas concretas, hombres y mujeres que no pueden desentenderse de su contexto social e histórico, de su religión, de sus ideas políticas o de su estatus económico con las más diversas motivaciones e intenciones, desde las más altruistas, como mejorar las condiciones de vida de la humanidad o adquirir un conocimiento más profundo de la Naturaleza, hasta las más profanas; como pueden ser aumentar su estatus o llenar sus bolsillos y no solo por el ideal de avanzar en la comprensión de la realidad. Por lo tanto, si la humanidad tiene una historia, la ciencia no ha de ser menos (Chade, 2014), sin poder comprenderse la una sin la otra.

En otro sentido, cuando, en el aula eliminamos estos condicionantes históricos o culturales desaprovechamos una magnífica ocasión para dotar de relevancia y significado a los nuevos conceptos, al establecer relaciones causa-efecto y ligando cada teoría con los problemas que llevaron a su elaboración (Campanario, 1998). Esto termina por provocar que el estudiante juzgue las aportaciones de los científicos que marcaron el devenir de la historia con valores y criterios actuales.

De esa forma, la supresión de las conexiones lógicas entre los conceptos, la problemática a la que dan respuesta y el contexto histórico en que se forjaron resulta en un aprendizaje arbitrario de la ciencia (Campanario, 1998), en el que no aparecen los límites de esta en la actualidad o las perspectivas de futuro que se mantienen abiertas, que acaba por cristalizar en una visión de la ciencia como cuerpo dogmático de conocimientos, incuestionables e inequívocos. Un conjunto cerrado de saberes con unos contenidos ya hechos, mostrados como las únicas conclusiones posibles, que además gozan de un valor propio, inherente.

No existe un cuerpo finito, terminado, de conocimientos científicos, sino un proceso dinámico de continua construcción y revisión de conceptos, leyes, teorías, hipótesis e interpretaciones (Martín et ál., 2002). Una vez tras otra planteamos preguntas a la naturaleza, descubriendo en ella aspectos que aún no conocemos, y a los que damos una respuesta construida de la mejor manera posible, pero nunca definitiva, siempre supeditada al surgimiento de nuevos interrogantes.

Una buena teoría no es una teoría absolutamente cierta e irrefutable, sino una explicación coherente y que posee una determinada eficacia en las condiciones dadas. Se trata, entonces, de valorarla en su contexto, considerando el influjo del entorno ideológico, religioso, social, etc. De acuerdo con Kuhn: 
La historia de la Ciencia no ha de buscar las contribuciones permanentes de una ciencia más antigua a nuestro conocimiento actual, sino que ha de poner de manifiesto la integridad histórica de esa ciencia en su época. No habrá que buscar, por ejemplo, la relación existente entre las opiniones de Galileo y las de la ciencia moderna, sino, más bien, la relación existente entre sus opiniones y las de sus maestros, contemporáneos y sucesores inmediatos. (1971, p. 23)

\section{Una imagen descontextualizada y} socialmente neutra de la actividad científica

La incuestionable supremacía del libro de texto en las aulas fomenta la creación de una imagen de la ciencia alejada y desconectada de la realidad, ajena a los problemas sociales (Vilches y Solbes, 2002), olvidando dimensiones esenciales de la actividad científico-tecnológica, como su componente humano o las complejas relaciones CTSA, las cuales quedan reducidas a la mera exposición de una serie de aplicaciones, sin tener en cuenta los intereses y la influencia de la sociedad en los descubrimientos científicos, el impacto que tuvieron y tienen en el medio natural o las repercusiones sociales que de ellos se derivaron en los diferentes momentos históricos (Fernández et ál., 2005). Se esconde así el papel que ha jugado la ciencia en la historia de la humanidad, su incidencia en nuestra concepción del mundo y sus repercusiones en la estructura de la sociedad y en la cultura (Vilches y Solbes, 2002).

Con frecuencia se obvian los graves conflictos que se han producido a lo largo de la historia y cómo han contribuido al progreso, a la dinamización, al avance de las disciplinas tecnocientíficas (Vilches y Solbes, 2002); cómo ese afán de dominación y de someter al ene- migo ha sido uno de los mayores motores del avance de la humanidad en materia de ciencia y tecnología. En consecuencia, aparece la falacia de la ciencia neutral, por encima de las ideologías, del bien y del mal, dedicada a la producción de conocimientos puros, objetivos e imparciales (Chade, 2014). Sin embargo, la realidad es que el avance de la ciencia dista de ser neutral junto con los incontables beneficios que ha logrado, también ha traído consigo consecuencias negativas.

Del mismo modo, aunque no debemos caer en la trampa de considerar la ciencia idílica, tampoco conviene abrazar la postura inversa, pecando de fatalistas y adhiriéndonos a las siempre peligrosas tendencias anticiencia. Posturas que cargan contra la ciencia y la tecnología, responsabilizándolas de la preocupante situación de deterioro que sufre nuestro planeta en los tiempos que corren, mientras ignoran convenientemente que son los científicos quienes estudian y se enfrentan a diario con los problemas que aquejan a nuestro planeta, quienes advierten de los riesgos y quienes trabajan con ahínco en la búsqueda de soluciones (Fernández et ál., 2002), pero que no son quienes tienen la potestad de legislar para proteger nuestro entorno.

Estas consideraciones suponen la admisión de que no pueden considerarse al margen de los condicionantes temporales, políticos, filosóficos, económicos, religiosos, morales, sociales y culturales en los que se han desarrollado, ya que es consecuencia y tiene implicaciones en todos ellos, pudiendo llegar a modificarlos, porque "enseñar conceptos fuera de su contexto supone falsificar la naturaleza del conocimiento científico y carece de toda utilidad, por mucho que parezca que se entienden dichos conceptos" (Lemke en Arroio, 2010).

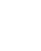


Por todo ello, es imprescindible que consigamos que la sociedad alcance a percibir la ciencia y la tecnología como un reflejo de la cultura y la época, una actividad comprometida con los valores, propiedades e intereses representativos de un contexto, contaminada por ellos y cuya evolución está ligada a sus designios e intereses; pero que, al mismo tiempo, y sin perjuicio de lo anterior las advierta como un proceso una construcción colectiva, un elemento cuya influencia y repercusión son claves en los grandes cambios sociales que han sido y que serán en el futuro.

\section{Una concepción exclusivamente analítica del conocimiento científico}

Una aproximación que incide con impertinencia en la necesaria parcelación y división de las disciplinas científicas (Fernández et ál., 2002), dejando de lado la gran importancia que tiene el enfoque multidisciplinar de los problemas, y las profundas interrelaciones e interdependencias que se establecen entre los distintos campos de estudio (Moreno, 2006). Es innegable que el desarrollo independiente de las disciplinas ha tenido aspectos muy positivos en todos los ámbitos, pero también ha traído consigo aspectos negativos, inconvenientes y trabas, como una excesiva y desmesurada superespecialización que compartimentaliza en demasía los saberes, alejándolos de la ciudadanía (Morin, 2001).

En la misma línea, se relegan al más absoluto ostracismo, los procesos de unificación, síntesis e integración parcial y progresiva del conjunto de conocimientos de que disponemos y el surgimiento de cuestiones puente entre disciplinas (Fernández et ál., 2002). Se olvida, con demasiada facilidad y frecuencia, que la reconstrucción didáctica es uno de los principales factores intrínsecos en la evolución del conocimiento (Moreno, 2006).

Se engloba aquí, además, la concepción excesivamente formalista, del conocimiento científico, que considera que la ciencia está constituida principalmente por artificios matemáticos que se han de aplicar mecánicamente a la resolución de problemas. En las aulas se dedica demasiado tiempo a la parte matemática, cuantitativa del proceso, la cual es de gran importancia, pero no es el componente esencial; se trata, más bien, de una herramienta, de un lenguaje que facilita la comprensión y la manipulación de los contenidos que realmente son vitales para la disciplina en cuestión. En cambio, el tiempo destinado al análisis del conocimiento y de las implicaciones que subyacen a desentrañar el misterio que continúa siendo para nosotros la naturaleza, se reduce a su mínima expresión.

Es cierto que el trabajo científico requiere una serie de tratamientos artificiales analíticos y simplificatorios (Fernández et ál., 2005). No obstante, cuando nos centramos en ellos y los convertimos en el eje del proceso, ponderándolos por encima de todo lo demás estamos perdiendo el norte, y mostrando una imagen que, además de sesgada, es falsa y resulta escasamente atractiva para el estudiante (Segarra et ál., 2017). 
Una visión meramente acumulativa del desarrollo científico

Fundamentada en una concepción del desarrollo científico marcada por un crecimiento lineal, acumulativo, con la incorporación sucesiva de nuevos conceptos y teorías que permiten ampliar de manera continua los límites del conocimiento (Fernández et ál., 2002). Aun así, el desarrollo científico está lejos de ser constante y acumulativo; más bien, es discontinuo, con sucesivas revoluciones y periodos de crecimiento exponencial, seguidos de etapas de profunda crisis, en las que parece que la actividad científica se estanca debido a su propio éxito, regodeándose en él.

La didáctica de las ciencias peca por omisión en este ámbito cuando presenta los conocimientos hoy aceptados sin considerar cómo han sido alcanzados, cuando ignora los momentos de profunda crisis, las remodelaciones y los cambios de paradigma que pusieron patas arriba las bases del conocimiento científico de la época (Fernández et ál., 2002). Cuando omite las controversias, las confrontaciones entre teorías, modelos y científicos y los pasos en falso que han dado lugar al progreso científico-tecnológico.

Aquí radica la imperiosa necesidad que tenemos de enseñar a nuestros estudiantes que el conocimiento tecnocientífico no es fijo e inamovible, no es una verdad absoluta y eterna, sino que se genera en un proceso dinámico, con pasos hacia delante y también retrocesos, pero siempre encaminado a la consecución de una comprensión más profunda de la realidad (Esteban, 2003). Las ideas científicas están sujetas al cambio, la ciencia no es estática, sino que constituye un panorama que cambia mientras lo observamos, a veces solo ligera, sutilmente, otras desdibujándose y difuminando sus límites y las artificiales líneas con las que pretendemos separar sus distintas ramas, mientras continúa, obstinada, con su proceso de producción de nuevos conocimientos.

Es nuestra obligación demostrarles que estos conocimientos son construcciones humanas, y para ello hemos de mostrarles los conceptos estructurantes que imperaban en los periodos de transformación profunda de las disciplinas, remarcando las características históricas, religiosas, políticas, económicas, filosóficas y sociales que entraron en juego, para hacerles entender que en cada momento los científicos empleaban las herramientas que tenían a su (Mora y Parga, 2010).

\section{Una imagen individualista y elitista de la ciencia}

En virtud de la cual parece que la ciencia avanza gracias los sucesivos descubrimientos realizados por abnegados eruditos, encerrados en laboratorios atestados de extraños instrumentos y que viven aislados del vulgo y alejados del mundanal ruido en sus torres de marfil (Fernández et ál., 2005). Superada ya la visión medieval del científico como un loco malvado parece que hoy la imagen es diametralmente opuesta (Acevedo, 2000). Ahora son considerados personas imprescindibles en la sociedad, brillantes, honradas y caracterizadas por su paciencia, su constancia y su determinación. Profesionales, honestos, meticulosos y minuciosos que viven al margen de los pormenores de la vida cotidiana (Gil y Vilches, 2001), dedicados en cuerpo y alma a la altruista tarea de buscar conocimientos para el beneficio social.

Esta concepción idealizada se ve enormemente reforzada por la opinión que impera acerca del saber científico y que deriva, principalmente, de las sensaciones y experiencias, por lo general negativas, que durante sus años escolares experimentaron muchos de quienes pasaron por las aulas. Se viene considerando 
a la ciencia y la tecnología como un conocimiento superior, restringido, esotéri$c o$, privilegio de unos pocos iniciados, e inalcanzable para la inmensa mayoría (Fernández et ál., 2002). Una parcela exclusiva y reservada para un número limitado de expertos dotados de la excepcional capacidad que constituyen la élite intelectual a la que solo las mentes más agudas y dotadas pueden aspirar.

El extremo rigor de la enseñanza tradicional, combinado con una visión predominantemente propedéutica (Furió et ál., 2001), y que destila sutilmente un cierto elitismo, de la didáctica de las ciencias, unido a su estigma de materias complejas y a la consiguiente normalización social y académica del fracaso en ellas, y, finalmente, su concepción excesivamente operativista, cristalizan en forma de una serie de expectativas negativas en el discente, que le persuaden de aproximarse a estas materias (Fernández et ál.,2005). Este distanciamiento se ve severamente agravado por un sistema educativo que criminaliza el error y que contribuye a esconder el carácter tentativo de las disciplinas científicas, su esencia como construcción humana y, por tanto, no exenta de equivocaciones, de las cuales el error forma parte intrínseca (Gil y Vilches, 2001), y se ha convertido en un medio inevitable para lograr los objetivos y afianzar la construcción de los distintos saberes, porque "el conocimiento es una aventura incierta que conlleva, en sí misma, permanentemente, el riesgo de ilusión y error [...], es navegar por un océano de incertidumbres a través de archipiélagos de certezas" (Morin, 2001).

Adicionalmente, se ignora el papel del trabajo en equipo, de intercambio de información, de la colaboración entre especialistas de diversas ramas. Se llega a hacer creer al alumno, incluso, que los hallazgos de un científico, un equipo de trabajo en el mejor de los casos, permite verificar o falsear una hipótesis o modificar una teoría (Fernández et ál., 2002). La realidad, en cambio, no podría ser más distinta, totalmente antitética, porque si hay algo que ha caracterizado a las especialidades científicas en el epílogo del siglo pasado y los prolegómenos del presente, es el incremento y la importancia, siempre crecientes, que han adquirido, continúan adquiriendo y adquirirán las dispares formas y procedimientos de integración horizontal (Cabot, 2014), de trabajo cooperativo, colaborativo y en equipo.

\section{Una visión androcéntrica de la ciencia}

Probablemente la que más desapercibida pasa, pues está integrada en la propia raíz de nuestra sociedad, extendiéndose a otros ámbitos de nuestra vida cotidiana. El arquetipo clásico de la persona dedicada a la investigación científica es un varón de mediana edad, caucásico, para más señas. Una iconografía que ha contribuido al mantenimiento de los arcaicos roles de género y que, en el ámbito académico, ha derivado en lo que los expertos denominan "visión de estereotipo" (Solbes et ál., 2007), que significa la admisión de una mayor 
dificultad a la hora de lidiar con determinadas asignaturas por la existencia de un estereotipo fijado en la sociedad.

El sistema tradicional de enseñanza de las ciencias ha sido en parte responsable de la expansión de esta concepción antropocéntrica. Diversas investigaciones (Solbes et ál., 2007; Vázquez y Manassero, 2008a, 2008b), ponen de manifiesto que los estudiantes muestran un claro desconocimiento de la carrera y las aportaciones de las científicas que ayudaron a la construcción y evolución del conocimiento científico. Se ha mostrado, y en muchos casos se sigue mostrando, un estereotipo de masculinidad, que pasa casi inadvertido o se produce por omisión y que, no obstante, ahí está, aunque sea de manera implícita, por ejemplo, en las ilustraciones de los libros de texto (Sánchez, 2002).

Los estudios de Vázquez y Manassero (2008a, 2008b) muestran que en general, en secundaria, los chicos tienen un mayor interés en ciencia y tecnología que sus compañeras y que, además, mientras ellas se inclinan más por la biología, ellos prefieren la física o la geología. Los resultados son todavía más preocupantes cuando se estudia la evolución de las actitudes de ambos géneros a medida que avanzan en el sistema educativo. Las chicas tienden a mostrar una mayor apreciación de la ciencia durante la primaria y comienzo de la secundaria, superior a la de sus compañeros. Sin embargo, este interés decrece mucho más rápido que el de ellos, especialmente en física, durante los años de instituto, muestra de la aceptación que se produce de los roles de género en esta etapa.

De esta forma, parece que todavía hoy existe una asociación más o menos implícita mujer-madre-cuidadora, que se traduce en la creencia de que el papel de la mujer en la ciencia ha de quedar restringido a los campos afines a la biología y a las ciencias de la salud, mientras que los físicos, los matemáticos, los ingenieros son los varones. En una etapa como la adolescencia, donde la sensación de pertenencia a un grupo incrementa exponencialmente su relevancia, donde la necesidad de encontrar un referente se hace más patente la didáctica de las ciencias fracasa al permitir la transmisión del estereotipo, al no aportar modelos a imitar a la mitad de los implicados en ella, a la mitad de la población.

\section{Conclusiones}

Desde el punto de vista del estudiante las emociones, las actitudes y la visión que tengan de la ciencia cobra una especial relevancia pues, llegado el momento, serán los elementos que determinen qué materias va cursar, qué itinerarios académicos e incluso qué orientación darles a sus futuros estudios. Por ello, la enseñanza de las ciencias, en su camino hacia la renovación para adecuar sus contenidos, lenguajes y métodos a las demandas de formación actuales y para alcanzar su meta de la formación de sujetos competentes en ciencias, que sepan, sepan hacer y sepan actuar, debe comenzar por abordar el problema de las visiones deformadas de la ciencia.

Partir de su análisis para diseñar un plan de acción con el que conseguir que nuestros jóvenes se acerquen a la verdadera naturaleza de la ciencia y la tecnología y comprendan el papel que ocupa tanto en nuestro día a día como en las complejas relaciones internacionales, para que sean conscientes de las libertades y comodidades de que gozamos gracias a sus avances.

Los docentes tenemos la obligación de proporcionar a nuestros jóvenes una visión más cercana y realista de la actividad científica, ofrecer una imagen de la ciencia como lo que es: una empresa humana, abierta y 
creativa, global, marcada por los valores, contingencias, vivencias y situaciones contextuales y personales que caracterizan a la cultura en que se genera y a las personas que la construyen, un medio para describir, explicar y comprender nuestro entorno y nuestra realidad, una herramienta para afrontar y resolver problemas.

En esta línea, la investigación en didáctica de las ciencias comienza a dar cada vez mayor importancia a la influencia educativa que tienen los concursos, ferias de ciencias y otras actividades de no formales, donde la ciencia y la tecnología se presentan al gran público de una manera informal y desenfadada. Así, se encuentran trabajos como los de Welch (2010) o Jones et ál. (2011), que concluyen que la participación en estos eventos mejora la percepción y la visión que los estudiantes tienen de las ciencias; y otros como, por ejemplo, los de Nájera (2010), Segarra et ál. (2008), Segarra et ál. (2017) que muestran directamente su utilidad para contribuir a la superación de las visiones deformadas.

En general, estos proyectos pretenden despertar el afán investigador de los participantes mediante el desarrollo de proyectos que los coloquen en la piel de auténticos investigadores, proponiendo hipótesis, buscando información y diseñando experimentos de laboratorio para contrastarlas, registrando observaciones y resultados en su cuaderno de laboratorio, analizándolos y extrayendo de ellos conclusiones que luego presentarán a sus compañeros y profesores, manejando un vocabulario científico riguroso, pero también a través de la conexión de los conocimientos con el mundo cotidiano del estudiante y su entorno.

\section{Agradecimientos}

Este trabajo es una contribución al grupo BEAGLE de investigación en Didáctica de la Ciencias Experimentales (Gobierno de Aragón). El autor disfruta de un contrato predoctoral del Gobierno de Aragón (ORDEN IIU/796/2019). El autor agradece a los revisores las sugerencias y propuestas realizadas para la mejora del trabajo.

\section{Referencias}

Acevedo, J. (2000). Una breve revisión de las creencias CTS de los estudiantes. Sala de lectura cts $+i$. Presentado en I Jornadas Universitarias de Nerva, Huelva.

Arraiz, A. y Sabirón, F. (2012). Orientación para el aprendizaje a lo largo de la vida: Modelos y tendencias. (Primera). Prensas de la Universidad de Zaragoza, D. L.

Arroio, A. (2010). Context based learning: A role for cinema in science education. Science Education International, 21 (3), 131-143.

Berbegal, A. (2015). Racionalidad y complejidad. http://personal.unizar.es/aberbegal/index.php/presentacion/apuntes-notas-y-borradores/ 
Borda, O. L. y Erazo, M. (2010). Concepciones sobre ciencia e investigación en profesores de química en formación inicial: Un estudio en el contexto de los trabajos. Tecné, Episteme y Didaxis: revista de la Facultad de Ciencia y Tecnología, 28, 41-56. https://doi. org/10.17227/ted.num28-1072

Cabot, E. A. (2014). Una aproximación a la concepción de ciencia en la contemporaneidad desde la perspectiva de la educación científica. Ciência y Educação (Bauru), 20(3), 549-560. https://doi.org/10.1590/151673132014000300003

Campanario, J. M. (1998). Ventajas e inconvenientes de la Historia de la Ciencia como recurso en la enseñanza de las ciencias. Revista de Enseñanza de la Física, 17 (0), 5-14.

Chade, P. O. (2014). Superación de las visiones deformadas de las ciencias a partir de la incorporación de la historia de la física a su enseñanza. Revista Eureka sobre enseñanza y divulgación de las ciencias, 17 (1), 34-53. https://doi.org/10.25267/Rev_Eureka_ensen_divulg_cienc.2014.v11.i1.05

Esteban, S. (2003). La perspectiva histórica de las relaciones Ciencia-Tecnología-Sociedad y su papel en la enseñanza de las ciencias. REEC: Revista electrónica de enseñanza de las ciencias, 2(3), 12.

Fernández, I., Gil, D., Carrascosa, J., Cachapuz, A. y Praia, J. (2002). Visiones deformadas de la ciencia transmitidas por la enseñanza. Enseñanza de las Ciencias, 20(3), 477-488.

Fernández, I., Gil Pérez, D., Valdés, P. y Vilches, A. (2005). ¿¿Qué visiones de la ciencia y la actividad científica tenemos y transmitimos? En D. Gil Pérez, B. Macedo, J. Martínez-Torregrosa, C. Sigifredo, P. Valdés, y A. Vilches (Eds.). ¿Cómo promover el interés por la cultura científica? Una propuesta didáctica fundamentada para la educación científica de jóvenes de 15 a 18 años (pp. 29-62). Oficina Regional de Educación de la Unesco para América Latina y el Caribe.

Furió, C., Vilches, A., Guisasola, J. y Romo, V. (2001). Finalidades de la enseñanza de las ciencias en la Secundaria obligatoria. ¿Alfabetización científica o preparación propedéutica? Enseñanza de las Ciencias, 19(3), 365-376.

Gallego, A. P. (2007). Imagen popular de la ciencia transmitida por los cómics. Revista Eureka sobre Enseñanza y Divulgación de las Ciencias, 4(1), 141-151.

García, I. F., Valdés, P., Pérez, D. G., Peña, A. V. y Sandoval, J. S. de. (2003). El olvido de la tecnología como refuerzo de las visiones deformadas de la ciencia. REEC: Revista electrónica de enseñanza de las ciencias, 2(3), 9.

Gil Pérez, D., Sifredo, C., Valdés, P. y Vilches, A. (2005). ¿ ¿Cuál es la importancia de la educación científica en la sociedad actual? En D. Gil Pérez, B. Macedo, J. Martínez-Torregrosa, C. Sifredo, P. Valdés, y A. Vilches (Eds.). ¿Cómo promover el interés por la cultura científica? Una propuesta didáctica fundamentada para la educación científica de jóvenes de 15 a 18 años (pp. 15-28). Oficina Regional de Educación de la Unesco para América Latina y el Caribe.

Gil Pérez, D. y Vilches, A. (2001). Una alfabetización científica para el siglo XXI: obstáculos y propuestas de actuación. Investigación en la Escuela, 43, 27-37.

Jones, G., Taylor, A. y Forrester, J. H. (2011). Developing a Scientist: A retrospective look. International Journal of Science Education, 33(12), 1653-1673. https:/1/doi.org/10.1 080/09500693.2010.523484

Kuhn, T. (1971). La estructura de las revoluciones científicas. Fondo de Cultura Económica.

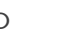


López, D. M. y Furió, C. (2017). Visiones deformadas de la ciencia en la enseñanza del concepto de elemento químico. Enseñanza de las ciencias: revista de investigación y experiencias didácticas, 0(Extra), 3747-3752.

Martín, M. J. (2002). Enseñanza de las ciencias ¿Para qué? Revista electrónica de enseñanza de las ciencias, 7 (2), 57-63.

Mora, W. M. M. y Parga, D. L. (2010). La imagen pública de la química y su relación con la generación de actitudes hacia la química y su aprendizaje. Tecné, episteme y didaxis: revista de la Facultad de Ciencia y Tecnología, (27), 67-93.

Moreno. M. (2006). El cine y la ciencia: Crónica de un desamor. Revista Mètode, 48. https://metode.es/revistas-metode/monograficos/el-cine-y-la-ciencia-cronica-de-un-desamor.html

Morin, E. (2001). Los siete saberes necesarios para la educación del futuro (Primera). Grupo Planeta.

Nájera, F. (2010). Los museos de ciencias, como una herramienta para superar algunos obstáculos epistemológicos de aprendizaje. Latin-American Journal of Physics Education, 4(Extra 1), 854-858.

Sánchez, A. (2002). El androcentrismo científico: El obstáculo para la igualdad de género en la escuela actual. Educar, 29, 91-102.

Segarra, A., Gadea, I., Vilches, A. y Gil Pérez, D. (2017). Uso de los museos para contribuir a superar las visiones deformadas de la ciencia. Enseñanza de las ciencias: revista de investigación y experiencias didácticas, O(Extra), 3827-3832-3832.

Segarra, A., Vilches, A. y Gil, D. (2008). Los museos de ciencias como instrumentos de alfabetización científica. Didáctica de las Ciencias Experimentales y Sociales, 22, 85-1 12. https://doi.org/10.7203/dces.2423

Solbes, J., Montserrat, R. y Furió, C. (2007). Desinterés del alumnado hacia el aprendizaje de la ciencia: Implicaciones en su enseñanza. Didáctica de las Ciencias Experimentales y Sociales, 21, 91-117. https://ojs.uv.es/index.php/dces/article/ view/2428

Vázquez, Á. y Manassero, M. A. (2008a). El declive de las actitudes hacia la ciencia de los estudiantes: Un indicador inquietante para la educación científica. Revista Eureka sobre Enseñanza y Divulgación de las Ciencias, 5(3), 274-292. https:// doi.org/10.25267/Rev_Eureka_ensen_divulg_cienc.2008.v5.i3.03

Vázquez, Á. y Manassero, M. A. (2008b). La química y el contexto de los estudiantes: El género y la primera elección de ciencias. Educación química, 19(4), 295-302. https://doi.org/10.22201/fq.18708404e.2008.4.25845

Vilches, A. y Solbes, J. (2002). Visiones de los estudiantes de secundaria acerca de las interacciones Ciencia, Tecnología y Sociedad. REEC: Revista electrónica de enseñanza de las ciencias, 1 (2), 3. 
Welch, A. (2010). Using the TOSRA to Assess High School Students' Attitudes toward Science after Competing in the FIRST Robotics Competition: An Exploratory Study. Eurasia Journal of Mathematics, Science and Technology Education, 6(3), 187-197. https://doi. org/10.12973/ejmste/75239

Forma de citar este artículo:

Martín-García, J. (2021). Nada es lo que parece: una reflexión sobre las visiones deformadas de la ciencia. Tecné, Episteme y Didaxis: TED, (50), 257 - 274. https://doi.org/10.17227/ ted.num50-9996 . 